\title{
Surgical Research
}

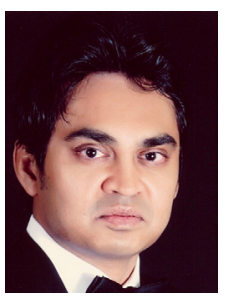

\section{The Subclavian Artery: What a Journey!}

\author{
Dr. Badr M I Abdulrauf MD FRCSC*
}

Section of Plastic and Reconstructive Surgery, Department of Surgery, King Faisal Specialist Hospital and Research Center Jeddah, Saudi Arabia.

${ }^{*}$ Correspondence:

Dr. Badr Abdulrauf MD FRCSC, Program Director-Plastic Surgery, PO Box 40047, MBC J-40, Jeddah 21499, Saudi Arabia.

Received: 12 April 2020; Accepted: 30 May 2020

Citation: Badr M I Abdulrauf. The Subclavian Artery: What a Journey!. Surg Res. 2020; 2(1): 1-2.

\begin{abstract}
The Subclavian artery represents the arterial supply system for the upper extremity. Its continuation under varying names with subdivisions, makes it such a significant vessel for numerous flaps through its many branches. A drawing summarizing these various segments with the five important landmarks which define those segments is portrayed. Such a concept would greatly facilitate a systematic studying process.
\end{abstract}

\section{Keywords}

Subclavian artery, Axillary artery, Brachial artery, Radial artery, Ulnar artery, Parts, Branches, Muscle flap, Fascio-cutaneous flap, Reconstruction, Systematic, Review, Studying.

"Education is what remains after one has forgotten what one learned in school"

\section{Albert Einstein}

\section{Concept}

Anatomy is probably one of the easily forgettable basic sciencs, this is why no other subject has the amount of mnemonics ever made compared to Anatomy [1].

The Subclavian artery (SA) is a unique vessel as it travels such a significant distance though under different names, before eventually bifurcating into two main, radial and ulnar arteries. SA is the basis for so many regional flaps whether in the neck, chest, breast, and the rest of upper extremity for many microvascular flaps. Besides that, SA or its continuity is often been operated upon purely as part of (Macro) vascular surgery.

When studying flaps, there is a need for systemization of one's thoughts by remembering the course of a major vessel and all the flaps it gives rise to. The SA artery in the upper part of the body is the best example to follow this principal.

\section{Image Analysis}

SA passes under different structures along its tract and it is being named differently after each of these stations, due to its lengthy pathway, passing in different territories. All the names it acquires indicate that specific region: Subclavian, Axillary, Brachial. The first two segments have different parts as well, first part of subclavian, second part and so on [2].

There are 5 important landmarks to remember: Scalene Anterior muscle; Outer border of first rib; Pectoralis minor muscle; Teres major muscle; and the neck of Radial bone. The SA $1^{\text {st }}$ part is medial to Scalene Anterior muscle, $2^{\text {nd }}$ part is behind the muscle and the $3^{\text {rd }}$ lateral to it. Once the vessel passes the outer border of $1^{\text {st }}$ rib it is named as Axillary. The Axillary artery's $1^{\text {st }}$ part is up to the Pectoralis minor muscle, $2^{\text {nd }}$ part is behind the muscle, and the $3^{\text {rd }}$ part lateral to it. Once the vessel passes the lower border of Teres major muscle it is named as Brachial artery.

At level of the Radial neck, it eventually divides into the Radial and Ulnar arteries (Figure 1).

Well known Muscle and Fasciocutaneous flaps dependent on the SA system include:

Pectoralis Major; Deltopectoral; Sternocleidomastoid; Trapezius; Latissimus dorsi; Serratus anterior; Scapular; Parascapular; Lateral arm; Radial and forearm flaps [3]. Furthermore, many branches from the SA system are considered major recipient vessels in 


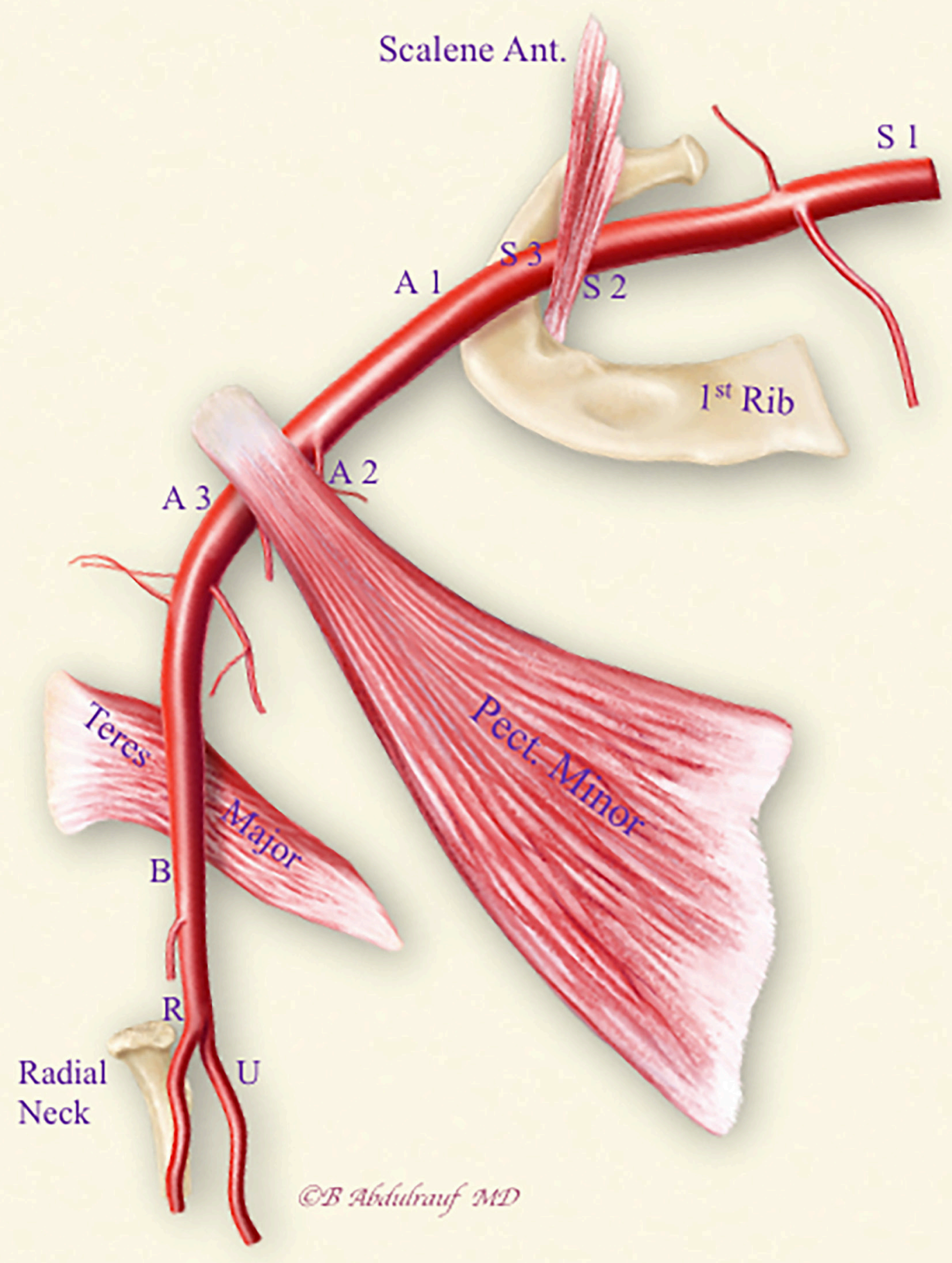

Figure 1: Author's drawing, depicting the course of SA, with its various segments and altering names, at specific anatomic points, up to the bifurcation into Radial and Ulnar arteries. S (Subclavian artery), A (Axillary artery) the numbers denote part of the artery; B (Brachial artery); R (Radial artery); U (Ulnar artery). The 5 important landmarks in relation to the main vessel, dividing it into various segments and parts are shown from proximal to distal: Scalene anterior muscle; First rib; Pectoralis minor muscle; Teres major muscle; and the Radial neck. It is up to the discretion of learner to name various branches from proximal to distal, and the known flaps they supply. 
microvascular transfers, like the internal mammary and lateral thoracic arteries.

It is a often noted that some vessels are named slightly differently in pure anatomy references versus operative technique ones. As an example, the Acromio-Thoracic artery which is a branch arising from the second part of Axillary artery [2] versus the ThoracoAcromial artery simply arising as branch off the Subclavian! [3].

Such issues can be a source of frustration to the learner, however probably it is a better idea to follow the standard anatomical teaching, so that there is consistency among all specialties. Besides of course understanding where is the branch located topographically, and its role in Pectoralis Major flap as an example. Entrapment syndromes, such as Subclavian steal, cervical rib and other pathologies such as vascular malformations can also involve SA system, requiring collaborative management.

\section{Discussion}

Anatomy other than being essential for the surgeon is "slippery" as well. We are all prone to forget important details. Here, we did not bring any new previously unknown anatomical knowledge as such, other than a viewpoint of how to keep "track" of many flaps based on branches of a Major vessel, (SA).

Another interesting vessel example in a different region, to follow a similar concept would be the External iliac artery. In- training residents in many surgical subspecialties and Interventional radiology are encouraged to use such ideas and illustrations as an image mnemonic, to help them recalling many relevant topics in their vast syllabus $[1,4]$.

In Plastic Reconstructive surgery where innovation and creativity are expected, a clear knowledge of anatomy is invaluable [5].

The SA with its long journey is a blessing to us as Reconstructive surgeons for the choices it provides, in solving significant number of problems.

\section{Summary}

A systematic approach for studying and reviewing flaps has been shown. The Subclavian artery is an ideal vessel to use such a concept, this was demonstrated in one single drawing. The students or residents in training are encouraged to use such a model in other regions as well.

\section{References}

1. Abdulauf BMI. A Mnemonic for Neck Triangles Anat. Physiol. 2020; 10: 323. doi:10.35248/2161-0940.2020.10.323

2. Gray's Anatomy The Anatomical basis of clinical practice 41st edition Elsevier.2015.

3. Grabb's EnFourth ecyclopedia of flaps dition. 2015.

4. Smith CF, Border S. The Twelve Cranial Nerves of Christmas: Mnemonics, Rhyme, and Anatomy - Seeing the Lighter Side. Anat. Sci Educ. 2019; 12: 673-677.

5. Rohrich RJ, Sullivan D. So you want to be like Leonardo da Vinci or Michelangelo? Which one are you? Plastic Reconstructive Surgery. 2011; 128: 1309-1311. 